\section{mCRC: Erhaltungstherapie und Lebensqualität}

\author{
Eine aktive Erhaltungstherapie kann die Wirksamkeit der Behandlung bei \\ Patienten mit metastasiertem kolorektalem Karzinom ( $m C R C$ ) erhöhen und \\ eine kürzere Induktionstherapie ermöglichen. Dies sollte aber mit mög- \\ lichst wenig Einbußen bei der Lebensqualität einhergehen.
}

\begin{abstract}
n der dreiarmigen Studie KRK 0207 der Arbeitsgemeinschaft Internistische Onkologie (AIO) wurde der Einfluss einer Erhaltungstherapie mit einem Fluoropyrimidin (FP) plus Bevacizumab (Arm A), mit Bevacizumab (BEV) alleine (Arm B) oder keiner Erhaltungstherapie (Arm C) auf die gesundheitsbezogene Lebensqualität (HR-QoL) untersucht. 837 Patienten hatten zunächst über 24 Wochen eine Induktion mit FP, Oxaliplatin und BEV erhalten, 472 konnten anschließend in einen der $3 \mathrm{Er}$ haltungstherapie-Arme randomisiert werden. Während der Induktions- und
\end{abstract}

Erhaltungstherapie wurde die HR-QoL alle 6 Wochen mit den EORTC-Fragebögen QLQ-C30 und QLQ-C29 erhoben. Hauptendpunkt war der mittlere Wert für die globale gesundheitsbezogene Lebensqualität (GHS/QoL) des EORTCQLQ-C30, kalkuliert als Durchschnittswert aller vorhandenen Erhebungen nach der Randomisierung.

Lebensqualitätsdaten standen von 413 Patienten zur Verfügung. Die Compliance mit den Fragebögen lag zu allen Zeitpunkten bei $\geq 95 \%$. Zu keinem Zeitpunkt ließ sich ein signifikanter Unterschied im mittleren GHS/QoL-Wert zwi- schen den 3 Armen feststellen. Auch die Analyse individueller Items der HR-QoL belegte keinen Unterschied.

Nach Meinung der Forscher spricht die fehlende Beeinträchtigung der Lebensqualität durch die Erhaltungstherapie für eine Sequenztherapie mit möglichst kurzer hochwirksamer Induktion und anschließender Erhaltung. Ein klarer Vorteil im Gesamtüberleben ist bisher allerdings nicht belegt.

Fazit: Die Fortsetzung der Behandlung durch eine aktive Erhaltungstherapie mit $\mathrm{FP} / \mathrm{BEV}$ beeinträchtigte bei mCRC-Patienten im Vergleich zu einer weniger intensiven oder keiner Erhaltung nicht die globale Lebensqualität. Friederike Klein

Quidde J et al. Quality of life assessment in patients with metastatic colorectal cancer receiving maintenance therapy after first-line induction treatment: a preplanned analysis of the phase III AIO KRK 0207 trial. Ann Oncol. 2016;27(12):2203-10.

\title{
Relevante geriatrische Faktoren bei mCRC
}

\section{Ein geriatrisches Assessment wird heute bei älteren Krebspatienten empfohlen. Welche Aspekte für Pro- gnose und Therapieentscheidung bei Patienten mit metastasiertem Kolorektalkarzinom (mCRC) relevant sind, untersuchten französische Onkologen.}

\footnotetext{
$\triangle \mathrm{n}$ der randomisierten Studie FFCD 2001-02 hatten zwischen Juni 2003 und Mai 2010282 über 75-jährige Patienten mit zuvor unbehandeltem mCRC teilgenommen. Sie erhielten 5-Fluorouracil (5-FU)-basierte Regime mit oder ohne Irinotecan (IRI). Ein geriatrisches Assessment war nicht verpflichtend, aber im Studienplan empfohlen. Entsprechende Daten vor Therapiebeginn lagen für 123 Patienten vor (62 mit 5-FU-, 61 mit 5-FUIRI-Therapie). Zu diesem Zeitpunkt betrug der Charlson-Komorbiditätsindex bei $75 \%$ der Patienten $\leq 1$, der Mini-Mental-State-Test war bei $31 \%$ leicht beeinträchtigt ( $\leq 27$ von 30 Punkten), der Wert auf der geriatrischen Depressionsskala
}

lag bei jedem zehnten Patienten über 2, und eine Beeinträchtigung in den instrumentellen Aktivitäten des täglichen Lebens (IADL) fand sich bei $34 \%$.

In der multivariaten Analyse war keiner dieser Parameter prädiktiv für objektive Ansprechrate (ORR) oder progressionsfreies Überleben (PFS). Ein Normwert im IADL war aber unabhängig von anderen Faktoren mit einem längerem $\mathrm{Ge}$ samtüberleben (OS) assoziiert. Eine Subgruppenanalyse wies (bei geringen Fallzahlen) auf einen möglichen Vorteil der Dublette bezüglich PFS bei einem Teil der Patienten hin. Faktoren, die eine Interaktion mit dem Behandlungseffekt von 5-FU-IRI zeigten, waren: Alter $\leq 80$ Jahre, unresezierter Primärtumor, Leukozytenzahlen $>11.000 \mathrm{~mm}^{3}$ und eine Erhöhung des Carcinoembryonalen Antigens (CEA) um mehr als das Doppelte. In der Subgruppe von Patienten mit IADLNormwert fand sich ein Trend hin zu einem OS-Vorteil.

Fazit: Die Autonomie im Alltag (IADL) scheint am ehesten ein unabhängiger

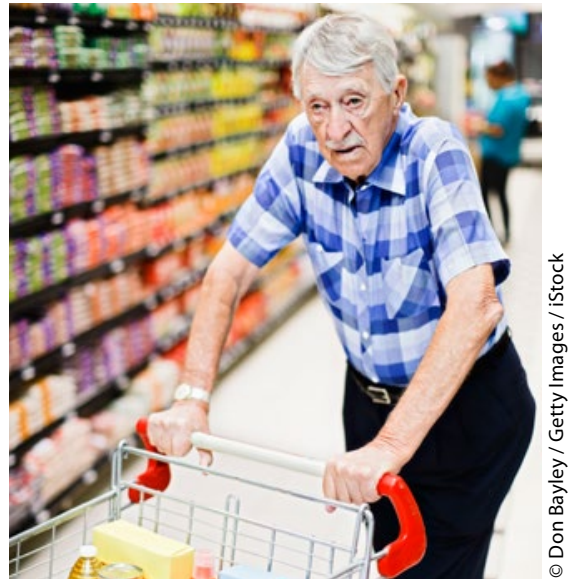

Die Alltagsautonomie könnte ein unabhängiger Faktor für das Überleben sein.

Prädiktor für das OS zu sein. Ein möglicher Vorteil der Dublette in einigen Subgruppen ist aufgrund der geringen Fallzahl und der widersprüchlichen Ergebnisse in anderen Studien mit Vorsicht zu interpretieren, eine Bestätigung in Studien ist notwendig. Friederike Klein

Aparicio T et al. Geriatric factors analyses from FFCD 2001-02 phase III study of first-line chemotherapy for elderly metastatic colorectal cancer patients. Eur J Cancer. 2017;74:98-108. 